\title{
Ovarian Hyperstimulation Syndrome in Natural Conception
}

\author{
${ }^{1}$ Sangeeta Pahwa, ${ }^{2}$ Sheena Chopra
}

\begin{abstract}
Background: Ovarian hyperstimulation syndrome (OHSS) generally results due to exogenous administration of gonadotropins for ovulation induction in females seeking treatment for infertility. OHSS can lead to life-threatening complications, therefore, its early diagnosis and management are very important.

Case report: We hereby report a rare case of OHSS in a spontaneously and naturally conceived pregnancy. Our patient is a 28-year old gravida 3 para 2 live 2 female with a period of gestation of 14 weeks, who presented to us with complaints of abdominal pain, mild distension, nausea, vomiting, and mild degree of breathlessness. All the possibilities that would have caused OHSS in a pregnancy were ruled out with adequate investigations. Ultrasound (USG)-guided aspiration of cyst was done and the cytology was negative for malignancy.

The patient and her attendants voluntarily demanded termination of pregnancy for the health interest of the mother. Termination of pregnancy was done. The postabortal period was uneventful. The follow-up scan after 4 weeks revealed bilateral ovaries near normal in size, shape, and volume. No ascites was found this time.

Conclusion: As after the termination of pregnancy B-human chorionic gonadotropin (HCG) levels dropped down and ovaries were found to be normal in the follow-up scan, our diagnosis goes more in favor of OHSS. Thus, although OHSS is a characteristic outcome of women who underwent some sort of ovarian induction or assisted reproductive technologies, one must be aware of its occurrence in a spontaneous conception too.
\end{abstract}

Keywords: Human chorionic gonadotropin, Ovarian hyperstimulation, Ovarian hyperstimulation syndrome, Spontaneous.

How to cite this article: Pahwa S, Chopra S. Ovarian Hyperstimulation Syndrome in Natural Conception. Curr Trends Diagn Treat 2018;2(2):109-111.

Source of support: Nil

Conflict of interest: None

\section{INTRODUCTION}

OHSS is almost invariably a complication of induction of ovulation. It generally results due to exogenous

\footnotetext{
${ }^{1}$ Professor, ${ }^{2}$ Junior Resident

1,2Department of Obstetrics and Gynaecology, Sri Guru Ram Das Institute of Medical Sciences \& Research, Amritsar, Punjab, India

Corresponding Author: Sangeeta Pahwa, Professor, Department of Obstetrics and Gynaecology, Sri Guru Ram Das Institute of Medical Sciences \& Research, Amritsar, Punjab, India, Phone: +919855080215, e-mail: sangeetadr30@gmail.com
}

administration of HCG for induction of ovulation in infertile subjects.

It is diagnosed early, as incidence of infertility is increasing nowadays and a large number of females are undergoing induction of ovulation and assisted reproductive technologies.

OHSS is classified as mild, moderate, severe, and critical. $^{1,2}$

It is characterized by ovarian enlargement with abdominal distension, ascites, hypotension, oliguria, hemoconcentration, and gastrointestinal and respiratory problems.

Its impact in severe forms can be fatal and life-threatening.

Spontaneous OHSS is extremely rare in naturally conceived pregnancies and only a few cases have been reported so far in the literature.

Spontaneous OHSS usually occurs at 8 to 14 weeks of the period of gestation because B-HCG peaks at around 10 weeks in natural conception, while iatrogenic OHSS usually occurs at 3 to 8 weeks of gestation as luteinizing hormone surge is achieved earlier with gonadotropin stimulation or with exposure to HCG. It is also observed after clomiphene citrate treatment or protocols in in vitro fertilization procedures.

\section{CASE REPORT}

We hereby report a case of a 28-year old female who is G3P2L2 (both alive). She presented, in the obstetrics outpatient department (OPD), with complaints of amenorrhea since three and a half months, abdominal pain, mild abdominal distension, nausea, vomiting, and breathlessness on exertion since 5 to 7 days.

Her past menstrual history was normal. No previous history of any medical/surgical illness present. The patient had previous two normal vaginal deliveries at term which were uneventful. Now, the patient conceived spontaneously and denied having taken any ovulation inducing agent.

On examination, the patient was calm, conscious, and well oriented. Her vitals were within normal limits. Pulse rate $(\mathrm{PR})=94 /$ minute, blood pressure $(\mathrm{BP})=120 / 80 \mathrm{~mm} \mathrm{Hg}$, respiratory rate $(R R)=16 /$ minute .

General physical examination was normal. The patient had mild pallor, no edema, cyanosis, or clubbing. Jugular venous pressure (JVP) was normal. Cardiovascular examination was normal.

On abdominal examination, the abdomen was soft, associated with diffuse tenderness. Bowel sounds were normal. Fundal height was 18 to 20 weeks of gestation 
(more than the period of amenorrhea). Fetal heart rate (FHR) was approximately 140 beats per minute.

She had a hemoglobin level of $11.2 \mathrm{~g} / \mathrm{dL}$ and hematocrit was $35.3 \%$.

Other routine investigations were within normal limits. Thyroid profile was normal. Viral markers were nonreactive.

ECG was normal.

Ultrasound for fetal well-being revealed gravid uterus with single gestational sac containing single live fetus corresponding to 14 weeks of gestation. Cardiac activity and somatic activity were normal. Placenta was anterior and amniotic fluid was adequate. No gross anomalies found (Fig. 1).

Bilateral ovaries were enlarged, hyperechoic, and smooth in outline. Right ovary $99 \times 78 \times 75 \mathrm{~mm}$ and about $307 \mathrm{cc}$ in volume. Left ovary $65 \times 52 \times 34 \mathrm{~mm}$ and $60.7 \mathrm{cc}$ in volume. Some vascularity seen and both ovaries mobile. Mild ascites with enlarged abdominal lymph nodes was seen.

Special investigations were done to rule out any malignancy. B-HCG = $52928 \mathrm{mIU} / \mathrm{mL}$, cancer antigen (CA) $125=160 \mathrm{U}$, alpha-feto protein $(\mathrm{AFP})=46 \mathrm{U}$.

Ultrasound-guided aspiration of cyst was done and sent for cytology which was negative for malignancy.

Due to the patient's history and laboratory findings, the diagnosis of spontaneous OHSS was considered. As the patient hading two live births, she and her attendants voluntarily demanded termination of pregnancy for the health interests of the mother.

Termination of pregnancy was done. Postabortal period was uneventful. Follow-up scan done after 4 weeks revealed that bilateral ovaries were normal in size and shape. The volume of the right ovary was reduced to $40 \mathrm{cc}$ and the left ovary to $20 \mathrm{cc}$. No ascites found.

\section{DISCUSSION}

OHSS is an extremely rare complication of spontaneous pregnancy usually occurring with high levels of HCG

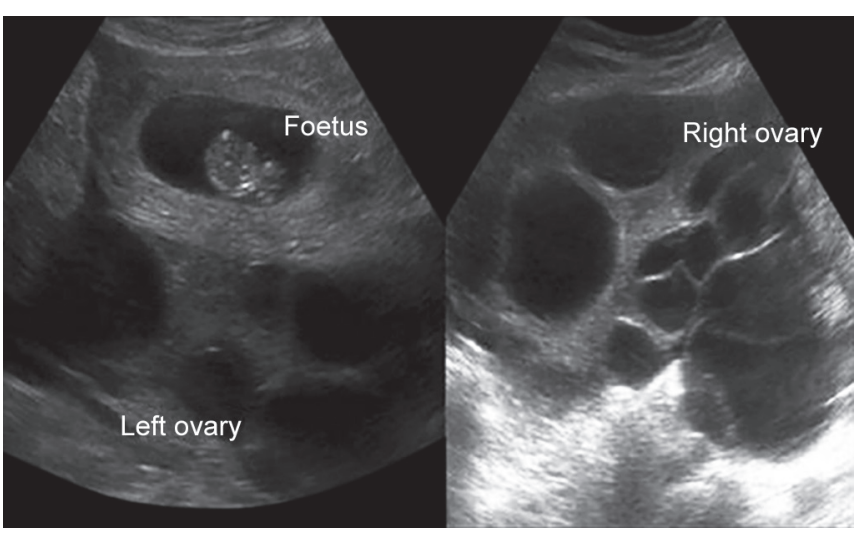

Fig. 1: Bilateral hyperstimulated ovaries with a single live fetus hormones. Severe OHSS is potentially a fatal complication of ovarian stimulation in assisted conception. It occurs in 0.2 to $1.2 \%$ of stimulated cycles.

It is characterized by enlargement of ovaries, increased vascular permeability, fluid shift from intravascular to extravascular space (abdomen, pleura, and pericardium), and ovarian neoangiogenesis which results in hemoconcentration, decreased renal clearance, oliguria/anuria, modification in coagulation factors, and thromboembolic risks.

Hemoconcentration leads to an increase of the hematocrit, the concentration of platelets and leucocytes, creatinine, ureum, and liver enzymes in the plasma, as well as hyperkalemia and acidosis. ${ }^{3}$

Primary risk factors for the development of OHSS include: young age, polycystic ovary syndrome (PCOS), lean body weight, high dose of gonadotropins, elevated estradiol and HCG levels, previous history of OHSS, molar pregnancy, multiple pregnancy, hypothyroidism, and high basal anti-Mullerian hormone levels.

Secondary risk factors are high or rapidly increasing estradiol levels, a high number of follicles, high vascular endothelial growth factor, and high inhibin B levels. ${ }^{4}$

In our case, there were no primary risk factors and all the causes that might have stimulated the ovaries were ruled out, like exogenous gonadotropins, HCG injections, and hypothyroidism.

This case presentation supports the idea that though exogenous gonadotropins and HCG are the most common causes of OHSS, it can also occur in naturally conceived singleton pregnancies.

Similarly, patients should be counseled, before starting assisted reproductive technology (ART) procedures, that iatrogenic complications can be associated with ovarian stimulation and/or oocyte aspiration. ${ }^{5}$

The syndrome is usually self limiting and continuation of pregnancy can be advised as B-HCG levels fall after first trimester. Since our patient was multipara with 2 live births, so, she opted for termination of pregnancy following which her symptoms improved.

Monitoring of hemodynamic status, intravenous crystalloid and albumin infusion, and prophylaxis of thrombosis are the main principles of management. ${ }^{6}$

Termination of pregnancy can be considered when conservative management fails, but surgery should be reserved for cases of ovarian rupture, torsion, and intraperitoneal hemorrhage.

\section{CONCLUSION}

As, after the termination of pregnancy, the patient recovered and ovaries were found to be near normal in 
the follow-up scan, our diagnosis goes more in favor of spontaneous OHSS.

To conclude, this case highlights several points:

- Though OHSS cases are commonly seen in patients undergoing in-vitro fertilisation (IVF), one must be aware of the occurrence of OHSS in spontaneous pregnancy.

- Early diagnosis is important and crucial for better and early management of the patient, thus reducing the maternal mortality rates.

\section{REFERENCES}

1. Ovarian Hyperstimulation Syndrome (OHSS) Diagnosis and Management. Institute of Obstetricians and Gynaecologists, Royal College of Physicians of Ireland and Directorate of Strategy and Clinical Programmes, Health Service Executive Version 1.0 April 2012 Guideline No. 9 Revision date: April 2014.
2. Shmorgun D, Claman P, Gysler M, et al. JOINT SOGCCFAS CLINICAL PRACTICE GUIDELINES COMMITTEE. The diagnosis and management of ovarian hyperstimulation syndrome. J Obstet Gynaecol Can 2011 Nov;33(11):1156-1162.

3. Ovarian Hyperstimulation Syndrome (OHSS) Diagnosis and Management. Institute of Obstetricians and Gynaecologists, Royal College of Physicians of Ireland and Directorate of Strategy and Clinical Programmes, Health Service Executive Version 1.0 April 2012 Guideline No. 9 Revision date: April 2014

4. Lodh M, Mukhopadhyay J, Sharma V. A case of severe ovarian hyperstimulation syndrome. Indian J Clin Biochem 2014 Jul;29(3):386-389.

5. Vloeberghs V, Peeraer K, Pexsters A, et al. Ovarian hyperstimulation syndrome and complications of ART [PubMed]. Best Pract Res Clin Obstet Gynaecol 2009 Oct;23(5):691-709.

6. Delbaere A, Smits G, Olatunbosun O, et al. New insights into the pathophysiology of ovarian hyperstimulation syndrome. What makes the difference between spontaneous and iatrogenic syndrome? Hum Reprod 2004 Mar;19(3):486-489. 